\title{
Caractéristiques dynamiques et thermiques d'un jet axisymétrique à propriétés variables : effet de la flottabilité
}

\author{
Ali Abbassia ${ }^{a}$, Najah Kechiche et Habib Ben Aissia \\ Unité de Métrologie en Mécanique des Fluides et Thermique, UMMFT 03/UR/11-09, Tunisie \\ École Nationale d'Ingénieurs de Monastir, Rue Ibn El Jazzar, 5019 Monastir, Tunisie
}

Reçu le 4 avril 2006, accepté le 2 novembre 2006

\begin{abstract}
Résumé - On propose, dans ce présent travail, des solutions numériques pour un écoulement stationnaire de type jet axisymétrique en régime laminaire. La variation des propriétés comme la masse volumique, la viscosité et la conductivité thermique avec la température est tenue en compte dans la formulation du problème. La variation du nombre de Prandtl avec la température est négligée. L'effet de l'écart de température entre le jet et le milieu ambiant est analysé en fonction du rapport des températures initiales $\Lambda$, pour les deux cas $\left(\Lambda=T_{0} / T_{\infty}>1\right.$ et $\left.\Lambda=T_{0} / T_{\infty}<1\right)$. L'effet des conditions d'émission (dites initiales) est aussi pris en compte dans cette étude, pour deux types de profils de vitesse et de température : uniforme et parabolique. La solution des équations régissant l'écoulement de type jet à propriétés variables est obtenue par une méthode aux différences finies. Les grandeurs analysées sont la vitesse et la température au centre, la vitesse et la température modifiées au centre, la demi-épaisseur dynamique et thermique du jet. Les deux régimes de convection mixte et forcée sont étudiés. Les résultats obtenus sont comparés à ceux proposés par d'autres auteurs, qui ont considéré deux contraintes d'intégration basées sur la conservation de la quantité de mouvement et de l'énergie qui remplacent, pour la résolution des équations, les conditions d'émission à la sortie de la buse. La comparaison des résultats obtenus par le présent modèle et ceux obtenus pour le cas où les propriétés sont prises constantes ainsi que ceux obtenus par d'autres auteurs est réalisée. Nos résultats et ceux proposés par ces auteurs montrent un accord satisfaisant uniquement dans la région loin de la buse, où les forces de flottabilité l'emportent sur les forces d'inertie.
\end{abstract}

Mots clés : Jet axisymétrique / régime laminaire / flottabilité / propriétés variables / convection forcée / convection mixte / vitesse au centre / température au centre / demi-épaisseur dynamique / demi-épaisseur thermique

\begin{abstract}
Dynamic and thermal characteristics of an axisymmetric jet with variable properties: Buoyancy effect. We propose in the present paper a numerical solution for a steady, laminar round jet flow with variable properties. Variation of properties like density, viscosity and thermal conductivity is considered in the formulation of the problem. Prandtl number variation with temperature is neglected. The effect of the initial temperature ratio $\Lambda=T_{0} / T_{\infty}$, between the jet and the surrounding medium, is analyzed for both cases $(\Lambda>1$ and $\Lambda<1)$. The effect of the initial velocity and temperature profiles is also taken into account. Two profiles are considered in the present work: uniform and parabolic, respectively. Solution of governing equations, of the buoyant jet flow with variable properties, is carried out by finite difference method on a staggered grid. Both forced and mixed convection regimes are investigated. The analyzed results are the centerline vertical velocity, the centerline modified velocity, the centerline temperature, the centerline modified temperature, the dynamical half-width and the thermal half-width. The results obtained are compared with those obtained by other authors, which replace the emission conditions at the nozzle exit by considering two constraints of integration: conservation of momentum and energy. Our results and those obtained by these authors are in good agreement only in the jet plume region when buoyant forces are responsible for flow. Results obtained by the present model and those obtained with constant properties models are also compared.
\end{abstract}

Key words: Round jet / laminar regime / buoyancy / variable properties / forced convection regime / mixed convection regime / centreline velocity / centreline temperature / dynamical half-width / thermal half-width

a Auteur correspondant : aaa_abbassi1973@yahoo.fr 


\section{Nomenclature}

\begin{tabular}{|c|c|}
\hline$C_{\mathrm{p}}$ & chaleur spécifique à pression constante, $\left[\mathrm{J} \cdot \mathrm{kg}^{-1} \cdot \mathrm{K}^{-1}\right]$ \\
\hline$D$ & diamètre de la buse circulaire, $[\mathrm{m}]$ \\
\hline$g$ & accélération de la pesanteur, $\left[\mathrm{m} . \mathrm{s}^{-2}\right]$ \\
\hline Gr & nombre de Grashof basé sur le diamètre de la buse $\left(G r=g \beta\left(T_{0}-T_{\infty}\right) D^{3} / \nu^{2}\right)$ \\
\hline $\operatorname{Pr}$ & nombre de Prandtl $\left(\operatorname{Pr}=\mu C_{\mathrm{p}} / \lambda\right)$ \\
\hline $\operatorname{Re}$ & nombre de Reynolds $\left(R e=U_{0} D / \nu\right)$ \\
\hline$R i$ & nombre de Richardson $\left(R i=G r / R e^{2}\right)$ \\
\hline$T$ & température, $[\mathrm{K}]$ \\
\hline$T_{0}$ & température à la sortie de la buse, $[\mathrm{K}]$ \\
\hline$u, v$ & composantes longitudinale et transversale de la vitesse, respectivement, $\left[\mathrm{m} . \mathrm{s}^{-1}\right]$ \\
\hline$U_{0}$ & vitesse initiale moyenne du jet, $\left[\mathrm{m} . \mathrm{s}^{-1}\right]$ \\
\hline$U_{\mathrm{m}}$ & vitesse modifiée \\
\hline$\theta_{\mathrm{m}}$ & température modifiée \\
\hline$U, V$ & composantes adimensionnées longitudinale et transversale de la vitesse, respectivement \\
\hline$x, y$ & coordonnées longitudinale et transversale, respectivement, [m] \\
\hline$X, Y$ & coordonnées adimensionnées longitudinale et transversale, respectivement \\
\hline$Y_{u}^{*}$ & demi-épaisseur dynamique \\
\hline$Y_{\theta}^{*}$ & demi-épaisseur thermique \\
\hline \multicolumn{2}{|c|}{ Symboles grecs } \\
\hline$\beta$ & coefficient de dilatation thermique, $\left[\mathrm{K}^{-1}\right]$ \\
\hline$\theta$ & température adimensionnée, $\left(\theta=\frac{T-T_{\infty}}{T_{0}-T_{\infty}}\right)$ \\
\hline$\Lambda$ & écart de température entre jet/ambiant $\left(T_{0} / T_{\infty}\right)$ \\
\hline$\mu$ & viscosité dynamique, $\left[\mathrm{kg} \cdot \mathrm{m}^{-1} \cdot \mathrm{s}^{-1}\right]$ \\
\hline$\lambda$ & conductivité thermique, $\left[\mathrm{W} \cdot \mathrm{m}^{-1} \cdot \mathrm{K}^{-1}\right]$ \\
\hline$\nu$ & viscosité cinématique, $\left[\mathrm{m}^{2} \cdot \mathrm{s}^{-1}\right]$ \\
\hline$\rho$ & masse volumique de l'air, $\left[\mathrm{kg} . \mathrm{m}^{-3}\right]$ \\
\hline$\Delta X, \Delta Y$ & pas de maillage dans les directions, $(X, Y)$ respectivement \\
\hline \multicolumn{2}{|l|}{ Indices } \\
\hline$c$ & valeur au centre du jet \\
\hline$m$ & grandeur modifiée \\
\hline 0 & valeur initiale à la sortie de la buse \\
\hline$\infty$ & valeur au milieu ambiant \\
\hline \multicolumn{2}{|c|}{ Exposants } \\
\hline$* *$ & valeur des propriétés en forme adimensionnées \\
\hline$*$ & correspond à la coordonnée transversale à laquelle $\left(U=U_{0} / 2\right)$ \\
\hline
\end{tabular}

\section{Introduction}

Les écoulements de type jets interviennent dans de nombreuses applications industrielles de grande importance, comme par exemple la pulvérisation, la climatisation, l'isolation thermique, le lissage des solides, la soudure, les chambres de combustion, etc. Les premiers travaux sur les jets datent depuis 1933, quand Schlichting [1] a proposé des solutions analytiques exactes concernant un jet axisymétrique. En 1937, Bickley [2] a publié une solution analytique pour un jet plan 2D. Les solutions proposées par Schlichting et Bickley sont basées sur les équations de la couche limite en régime laminaire. Le résultat de ces analyses montrent que la vitesse axiale décroît en fonction de la distance longitudinale $X$ mesurée à partir de la sortie de la buse. Martynenko et al. [3] présentent une analyse détaillée des écoulements de type jets en tenant compte des effets de la flottabilité, en utilisant l'approximation de Boussinesq. L'étude effectuée par Martynenko et al. [3] ignore l'effet des conditions d'émission sur la dynamique du jet en utilisant deux contraintes d'intégration qui sont la conservation de la quantité de mouvement et du flux de chaleur. En effet, ces deux contraintes remplacent les conditions d'émission à la 
sortie de la buse et peuvent être vérifiées, quelle que soit la forme des profils de vitesse et de température initiaux. Yu et al. [4] présentent des solutions rigoureuses pour la zone intermédiaire, où les forces d'inertie et de flottabilité sont du même ordre de grandeur. Ils ont utilisé un changement de variables et ont introduit de nouveaux paramètres, leurs solutions ignorent l'effet des conditions d'émission et la dépendance des propriétés avec la température sur la structure de l'écoulement.

En résumé, la plupart des travaux effectués sur ce type d'écoulement en régime laminaire [1-12] se limitent à des solutions analytiques ou numériques des équations de conservation, en utilisant un changement de variables qui permet d'ignorer les conditions d'émission à la sortie de la buse. Pour les jets chauffés, plusieurs études ont traité, pour le cas laminaire, uniquement les deux zones extrêmes dans la direction de l'écoulement (existence de solutions similaires) : la zone de panache (loin de la buse où les forces de flottabilité dominent l'écoulement) [3-9] et la région du jet (près de la buse, où le jet est essentiellement gouverné par les forces d'inerties) [1]. L'étude de Yu et al. [4] pour le cas d'un jet plan et celle de Martynenko et al. [3] pour le cas d'un jet axisymétrique proposent des solutions qui sont valables pour les différentes zones du jet.

Malgré la vaste littérature consacrée à l'étude de l'écoulement de type jet, en régime laminaire ou turbulent, il reste des effets, tels que par exemple l'effet des conditions d'émission, la transition du jet du régime de convection forcée au régime de convection mixte et la variation des propriétés du fluide avec la température, qui n'ont pas été explorés en détails. Ben Aissia et al. [10,11] ont mené une étude numérique concernant un jet axisymétrique chauffé en tenant compte de l'effet des conditions d'émission à la sortie de la buse. Ces auteurs se basent dans leur étude, pour des nombres de Reynolds allant de 250 à 1500, sur les équations de la couche limite laminaire et stationnaire avec les approximations de Boussinesq pour la modélisation du terme de flottabilité $[10,11]$. Ils montrent que, l'effet de la flottabilité est important pour des distances longitudinales $X$ loin de la sortie de la buse (zone de panache), et qu'au contraire, les conditions d'émission contrôlent l'écoulement dans la zone du jet ( $X$ proche de la sortie de la buse). Ces auteurs ne tiennent pas compte de la variation des propriétés avec la température sur l'évolution des propriétés dynamiques et thermiques du jet.

Dans les processus thermiques et hydrodynamiques, qui se produisent dans les applications industrielles, la plupart des écoulements sont à propriétés variables, dues à l'existence d'un fort écart de température ou à des réactions chimiques dans l'écoulement. Les écoulements de type jet qui interviennent dans les applications industrielles sont turbulents et à propriétés variables. Plusieurs études expérimentales ont été effectuées pour déterminer le comportement d'un jet axisymétrique dans différentes zones et d'étudier le passage de l'écoulement d'une zone à l'autre sous l'effet des forces de flottabilité [13-16]. En effet, l'intensité des forces de flottabilité vis-à-vis des forces d'inertie (mesurée par le nombre de Richardson Ri), peut faire passer l'écoulement du régime de jet inertiel à celui de panache à travers un régime intermédiaire : le régime transitoire. Sanders et al. $[17,18]$ proposent des solutions numériques d'un jet axisymétrique à densité variable en régime turbulent en utilisant un modèle de fermeture du premier et du second ordre et montrent que le modèle de fermeture du second ordre donne des résultats qui coïncident mieux avec les résultats expérimentaux.

Dans cet article, on propose des solutions numériques pour un écoulement stationnaire de type jet axisymétrique à propriétés variables, en régime laminaire, en tenant compte des conditions d'émission à la sortie de la buse, tout en s'assurant que les deux contraintes d'intégration (conservation de la quantité de mouvement et de l'énergie), adoptées par Martynenko et al. [3] soient vérifiées. On s'intéresse essentiellement à l'analyse de l'effet de l'écart de température entre le jet et le milieu ambiant, en fonction du rapport des températures initiales $\Lambda=T_{0} / T_{\infty}$, sur les caractéristiques thermiques et dynamiques du jet dans les deux régimes de convection : forcée et mixte.

\section{Hypothèses}

On considère un écoulement stationnaire de type jet axisymétrique qui est immergé dans un milieu non-confiné constitué du même fluide, à températures différentes. La variation de la masse volumique avec la température obéit aux approximations de Boussinesq dans le terme de flottabilité, tout comme pour le cas où les propriétés sont prises constantes [19]. La variation du nombre de Prandtl et de la chaleur massique avec la température est négligée. Les nombres de Reynolds considérés sont suffisamment élevés, par conséquent la couche de cisaillement du jet est mince. L'épaisseur de la couche de cisaillement est beaucoup plus petite que la dimension longitudinale de l'écoulement. En outre, la composante axiale $u$ de la vitesse est beaucoup plus grande que la composante radiale $v$, par conséquent les approximations de la couche limite sont applicables. L'écoulement est à nombre de Mach inférieur à 1. La pression est hydrostatiquement distribuée à travers le jet. L'expérience montre toutefois qu'il y a une petite variation de la pression statique dans la zone du noyau potentiel $[20]$.

\section{Modèle mathématique et méthode numérique}

\section{1 Équations adimensionnées}

D'après les hypothèses précédemment citées, ce sont les équations de la couche limite qui formulent l'écoulement stationnaire, laminaire et bidimensionnel de type jet à propriétés variables. Ces équations ainsi que leurs conditions aux limites et initiales sont mises sous 


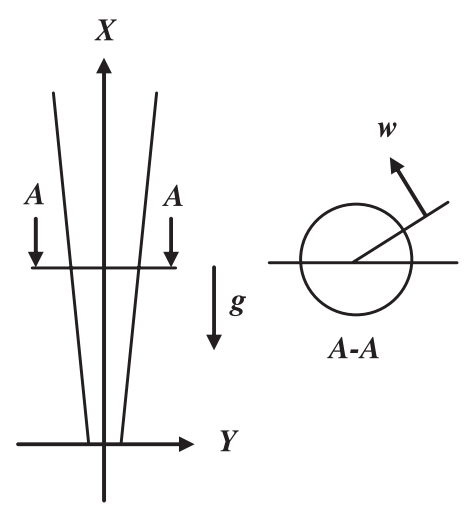

Fig. 1. Schéma de la buse d'injection.

forme adimensionnée, en considérant les variables adimensionnelles suivantes :

$$
\begin{aligned}
& U=u / U_{0}, \quad V=v / U_{0}, \quad \theta=\left(T-T_{\infty}\right) /\left(T_{0}-T_{\infty}\right) \\
& X=x / D, \quad Y=y / D, \\
& \rho^{* *}=\rho / \rho_{\infty}, \quad \mu^{* *}=\mu / \mu_{\infty}, \quad \lambda^{* *}=\lambda / \lambda_{\infty}
\end{aligned}
$$

Les équations régissant l'écoulement de type jet à propriétés variables, peuvent alors s'écrire sous la forme suivante, en coordonnées cylindriques en utilisant les nouvelles variables adimensionnelles (Éq. (1)) :

Équation de conservation de la masse :

$$
\frac{\partial\left(\rho^{* *} Y U\right)}{\partial X}+\frac{\partial\left(\rho^{* *} Y V\right)}{\partial Y}=0
$$

Équation de la quantité de mouvement :

$$
\rho^{* *} U \frac{\partial U}{\partial X}+\rho^{* *} V \frac{\partial U}{\partial Y}=\frac{1}{\operatorname{Re} Y} \frac{\partial}{\partial Y}\left(\mu^{* *} Y \frac{\partial U}{\partial Y}\right) \pm \epsilon R i \rho^{* *} \theta
$$

Équation de l'énergie :

$$
\rho^{* *} U \frac{\partial \theta}{\partial X}+\rho^{* *} V \frac{\partial \theta}{\partial Y}=\frac{1}{\operatorname{RePr} Y} \frac{\partial}{\partial Y}\left(\lambda^{* *} Y \frac{\partial \theta}{\partial Y}\right)
$$

où $\epsilon$ dans l'équation de quantité de mouvement (Éq. (3)) prend les valeurs 0 et 1 . $\epsilon=0$ correspond au cas d'un écoulement de type jet isotherme et par conséquent les deux problèmes dynamique et thermique sont découplés. Pour le cas non-isotherme, $\epsilon=1$, le signe + représente le cas où le rapport de température $T_{0} / T_{\infty}>1$ alors que le signe - représente celui où le rapport de température $T_{0} / T_{\infty}<1$. Ces équations sont écrites dans un système de coordonnées cylindriques tel que l'origine des axes se trouve au milieu de la section de sortie de la buse (Fig. 1).

Aux équations de conservation (Éqs. (2)-(4)) sont associées les conditions aux limites suivantes, sous forme adimensionnées :

$$
\begin{array}{ll}
Y=0 ; & \frac{\partial U}{\partial Y}=0, \quad \frac{\partial \theta}{\partial Y}=0, \quad V=0 \\
Y \rightarrow \infty ; \quad U \rightarrow 0, \quad \theta=0
\end{array}
$$

En plus de ces conditions aux limites, Martynenko et al. [3] ont considéré deux contraintes d'intégration : i) conservation de la quantité de mouvement (Éq. (3)), ii) conservation de l'énergie.

L'énergie transportée par l'écoulement pour $x>0$ est une constante égale à la valeur de l'énergie déchargée par la buse, qui s'écrit pour un jet libre à propriétés physiques constantes :

$$
2 \pi \rho C_{\mathrm{p}} \int_{0}^{\infty} u\left(T-T_{\infty}\right) y \mathrm{~d} y=Q_{0}
$$

où $Q_{0}$ représente le taux de flux de chaleur initiale déchargée par la buse. La seconde contrainte provient de la conservation de la quantité de mouvement (Éq. (3)). L'intégration de l'équation de la quantité de mouvement, pour un jet libre à propriétés physiques constantes, donne :

$$
\frac{\mathrm{d}}{\mathrm{d} x} \int_{-\infty}^{\infty} u^{2} y \mathrm{~d} y=\int_{-\infty}^{\infty} g \beta\left(T-T_{\infty}\right) y \mathrm{~d} y
$$

Dans la région très proche de la buse $(x \rightarrow 0)$, les forces de flottabilité sont négligeables devant les forces d'inertie, et l'équation (Éq. (7)) devient alors,

$$
K_{0}=\lim _{x \rightarrow 0} \int_{0}^{\infty} 2 \pi \rho u^{2} y \mathrm{~d} y=\text { const. }
$$

où $K_{0}$ représente le taux de flux initial de la quantité de mouvement à la sortie de la buse.

Les conditions initiales adimensionnées, de vitesse et de température, pour le jet, s'écrivent de la façon suivante :

$X=0 ; \quad V=0 \quad$ et $:$

$$
\begin{cases}\bullet 0<Y<0,5 & \\ \text { Profil uniforme : } & U=1 ; \theta=1 \\ \text { Profil parabolique : } & U=(3 .)^{0,5}\left(1-4 Y^{2}\right) ; \\ & \theta=(3 .)^{0,5}\left(1-4 Y^{2}\right) \\ \bullet Y>0,5 & \\ U=0 ; \theta=0 . & \end{cases}
$$

Les constantes qui figurent dans les profils initiaux de vitesse et de température sont déterminées de façon à ce que les constantes d'intégration (conservation de la quantité de mouvement et de l'énergie) proposées par Martynenko et al. [3] soient vérifiées. Les équations adimensionnées (Éqs. (2)-(4)) et leurs conditions aux limites (Éq. (5)) et initiales (Éq. (9)) sont résolues par une méthode aux différences finies utilisant un maillage $(2 \mathrm{D})$ décalé, montré sur la figure 2 . Les indices $(i, j)$ représentent les directions $X$ et $Y$, respectivement. Le point $A$ représente le point de coordonnées $X=0$ et $Y=0$. L'indice $(0,0)$ représente ce point (centre de la buse d'injection). 


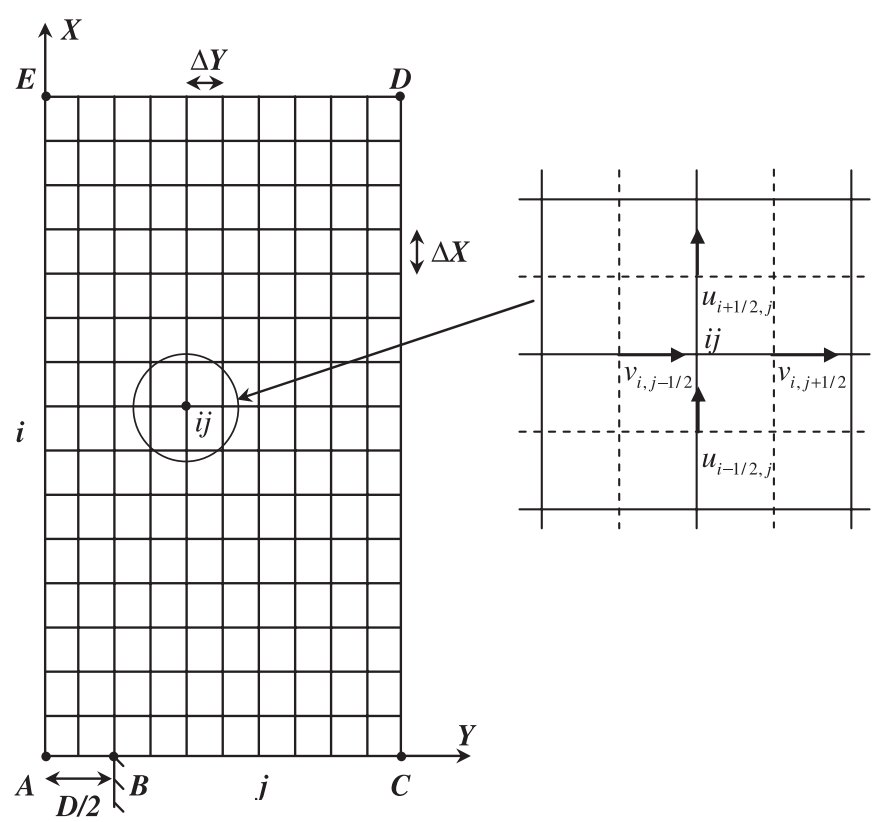

Fig. 2. Schéma du maillage, sur le demi-espace du domaine de calcul.

L'incrémentation suivant la direction $X$ (i.e., $X+\Delta X$ ) est $i+1$, et suivant la direction $Y$ (i.e., $Y+\Delta Y$ ) est $j+1$. Les indices $i, j$ parcourent les nœuds de $0, \ldots, I_{\max }$ et $0, \ldots, J_{\max }$, respectivement.

Les équations de quantité de mouvement (Éq. (3)) et d'énergie (Éq. (4)) sont discrétisées aux nœuds $(i+1 / 2, j)$. Pour améliorer la précision, nous avons utilisé une méthode aux différences finies centrées suivant les deux directions $X$ et $Y$. L'erreur de troncature de cette méthode est de $O\left(\Delta X^{2}, \Delta Y^{2}\right)$. Ceci donne une précision de second ordre suivant $X$ et $Y$ pour la vitesse longitudinale $U$ et la température $\theta$. L'équation de continuité (Éq. (2)) est discrétisée aux nœuds $(i+1 / 2, j+1 / 2)$. Une méthode aux différences finies centrées est aussi appliquée à cette équation ce qui donne une erreur de troncature de l'ordre $O\left(\Delta X^{2}, \Delta Y^{2}\right)$, et une solution du second ordre pour la composante transversale $V$ de la vitesse.

La forme discrétisée, des équations de conservation, utilisée dans ce présent travail est similaire à celle utilisée par Dalbert et al. [21], elle a été adoptée pour des raisons de stabilité numérique par rapport à un maillage nondécalé.

\section{Conditions aux limites}

Le calcul se fait ligne par ligne (i.e. $i=0, \ldots, I_{\max }$ ). Le jet étant symétrique par rapport à l'axe $O X$, par conséquent on ne considère que le demi-espace du jet. Dans la direction $O Y$, le pas de calcul impose un nombre suffisant de points $n$ dans cette direction pour que le jet ne soit pas coupé. La distance $Y_{\infty}=(n-1) \Delta Y$, pour les nombres de Reynolds et de Richardson utilisés, est de 16. Cette distance permet une bonne approximation pour les conditions aux limites de type Dirichlet (pour $Y \rightarrow \infty$ ) utilisées dans ce travail (Éq. (5)). (a) Pour $X=0$, sortie du jet (i.e. $i=0$ ), on initialise les champs de vitesse et de température par les profils initiaux (uniforme ou parabolique (Éq. (9))).

(b) Pour $Y=0$, nous avons utilisé une méthode aux différences finies, pour les conditions aux limites de type Neumann sur la vitesse et la température (Éq. (5)). Les équations de la quantité de mouvement (Éq. (3)) et de l'énergie (Éq. (4)) deviennent, respectivement :

$$
\begin{array}{r}
\left(\rho^{* *} U \frac{\partial U}{\partial X}\right)_{i j=0}=\frac{1}{R e}\left(\mu^{* *} \frac{\partial^{2} U}{\partial Y^{2}}\right)_{i j=0} \pm \epsilon R i\left(\rho^{* *} \theta\right)_{i j=0} \\
\left(\rho^{* *} U \frac{\partial \theta}{\partial X}\right)_{i j=0}=\frac{1}{R e P r}\left(\lambda^{* *} \frac{\partial^{2} \theta}{\partial Y^{2}}\right)_{i j=0}
\end{array}
$$

Le maillage utilisé est uniforme, le pas de calcul suivant la direction longitudinale et transversale étant constant. En effet, le pas de calcul est pris égal à $\left(\Delta X=10^{-3}, \Delta Y=10^{-3}\right)$, suivant les directions $X$ et $Y$, respectivement. La résolution spatiale et le test de convergence de cette méthode ont été validés par Ben Aissia et al. $[10,11]$. La solution du système algébrique tridiagonal est obtenue en utilisant l'algorithme de Thomas [22]. La solution pour les variables $U, V$ et $\theta$ est obtenue progressivement en commençant à partir de $X=$ 0 . Premièrement, l'équation de quantité de mouvement (Éq. (3)) est résolue pour obtenir la valeur de la composante longitudinale $U$ de la vitesse, en utilisant un champ de vitesse arbitraire que nous avons choisi égal à la vitesse initiale sur la largeur de la buse et nul ailleurs (Éq. (9)). Ensuite, l'équation de continuité (Éq. (2)), est résolue en utilisant la valeur de la vitesse $U$, obtenue à partir de l'équation de la quantité de mouvement (Éq. (3)), pour obtenir la valeur de la composante transversale $V$ de la vitesse. Les valeurs des composantes des vitesses $U$ et $V$ sont ensuite injectées dans l'équation de l'énergie (Éq. (3)) pour obtenir la température $\theta$. Les propriétés physiques $\left(\rho^{* *}, \mu^{* *}\right.$, et $\left.\lambda^{* *}\right)$ sont enfin calculées en utilisant le champ de température qui vient d'être calculé (Éq. (12)) [19]

$$
\begin{array}{ll}
\text { Densité } & : \rho^{* *}=[1+\theta(\Lambda-1)]^{-1} \\
\text { Viscosité } & : \mu^{* *}=[1+\theta(\Lambda-1)]^{0,71} \\
\text { Conductivité } & : \lambda^{* *}=[1+\theta(\Lambda-1)]^{0,85}
\end{array}
$$

La procédure itérative consiste à résoudre, l'équation de la quantité de mouvement, de continuité et de l'énergie dans cet ordre, répétitivement jusqu'à ce que la convergence soit atteinte. La convergence de la solution globale est obtenue lorsque le changement relatif de la vitesse $U$ lors de deux itérations successives est inférieur à $10^{-6}$ pour chaque nœud du domaine de calcul $[10,11]$.

Les valeurs convergées de $U, V$ et $\theta$, obtenues pour $j=$ 0 jusqu'à $j=J_{\max }$ pour la même ligne $i$ sont utilisées pour obtenir les valeurs de ces variables pour la ligne suivante (i.e., $i+1)$, la procédure sera terminée lorsque la dernière ligne $I_{\max }$ du domaine est atteinte. Afin de comparer nos 
résultats avec ceux obtenus par Martynenko et al. [3], le même code a été utilisé, avec quelques modifications, pour le cas d'un jet chauffé à propriétés physiques constantes.

Pour tous les nombres de Reynolds et de Richardson utilisés pour le calcul numérique du problème de jet axisymétrique à propriétés variables, présenté dans ce travail, nous avons calculé, pour différentes distances longitudinales $X$, la quantité de mouvement, afin de nous assurer qu'il y avait bien conservation de cette grandeur (deuxième contrainte d'intégration de Martynenko et al. [3]). La contrainte d'intégration basée sur la quantité de mouvement pour un jet à propriétés physiques constantes est, d'après l'équation (Éq. (8)) :

$$
K_{0} / 2 \pi \rho=\int_{0}^{\infty} u^{2} y \mathrm{~d} y=\text { const. }
$$

\section{Résultats et discussions}

Le modèle numérique que nous avons développé, nous a permis de déterminer les caractéristiques dynamiques et thermiques d'un jet axisymétrique à propriétés variables pour les deux régimes de convection : forcée et mixte. L'influence de l'écart de température entre le jet et le milieu ambiant sur la structure du jet est analysée en étudiant, en fonction du rapport des températures initiales $\Lambda=T_{0} / T_{\infty}$ :

- la vitesse verticale au centre $U_{\mathrm{c}}(Y=0)$ dans les deux régimes de convection;

- la vitesse verticale modifiée au centre $U_{\mathrm{cm}}$;

- la température au centre $\theta_{\mathrm{c}}(Y=0)$ dans les deux régimes de convection;

- la température modifiée au centre $\theta_{\mathrm{cm}}$;

- la demi-épaisseur dynamique $Y_{u}^{*}$ (définie comme étant l'ordonnée $Y$ correspondante à $\left.U_{\mathrm{c}} / 2\right)$;

- la demi-épaisseur thermique $Y_{\theta}^{*}$ (définie comme étant l'ordonnée $Y$ correspondante à $\left.\theta_{\mathrm{c}} / 2\right)$;

Il faut noter qu'on traite uniquement dans ce travail, le cas d'un jet favorable, de sorte que les forces de flottabilité sont dirigées dans le sens de l'accélération de la pesanteur,(i.e., pour $\Lambda>1$ le jet est ascendant (Fig. 1), alors que pour le cas $\Lambda<1$ le jet est descendant). Pour valider le modèle numérique présenté dans cet article, les résultats sont comparés à ceux obtenus par d'autres auteurs et à ceux obtenus dans le cas où les propriétés sont prises constantes.

\subsection{Régime de convection forcée}

\subsubsection{Vitesse et température au centre}

Dans cette section, on s'intéresse à l'analyse de l'effet de l'écart de température $\Lambda$ (rapport des températures initiales) entre le jet et le milieu ambiant, sur le comportement dynamique et thermique d'un jet d'air chauffé, $\operatorname{Pr}=0,71$, dans le régime de convection forcée, pour un nombre de Richardson, $R i=5 \times 10^{-4}$. L'effet du profil de la vitesse à la sortie de la buse et du nombre de Reynolds est également analysé. Les résultats obtenus par le présent modèle sont comparés à ceux obtenus par Martynenko et al. [3] et à ceux obtenus par un modèle à propriétés constantes.

En ce qui concerne la vitesse verticale au centre $U_{\mathrm{c}}$, nous remarquons, d'après la figure $3 \mathrm{a}$, pour un écart de température $\Lambda=0,75$, que pour un profil initial uniforme (trait interrompu) et pour les faibles distances longitudinales $X$, la vitesse verticale au centre du jet conserve une valeur constante, égale à celle de la sortie de la buse, ce qui montre l'existence d'un noyau potentiel. Par ailleurs, en s'éloignant de la buse, on constate que la vitesse au centre décroît plus rapidement pour les faibles nombres de Reynolds, pour la même distance longitudinale $X$. Pour le cas du profil initial parabolique (trait continu), la vitesse au centre ne présente pas une partie constante, pour les faibles distances $X$. En effet, elle décroît dès la sortie de la buse. Le jet ne présente pas un noyau potentiel pour ce cas du profil initial (parabolique) $[11,12,20]$. La différence entre la vitesse au centre, pour les deux cas de profils initiaux de vitesses, est très importante uniquement dans la région proche de la buse : zone du jet. En effet, dans cette région le jet est gouverné essentiellement par les forces d'inertie. Pour les distances longitudinales $X$ très élevées, l'évolution de la vitesse centrale est identique, quel que soit le profil initial de la vitesse à la sortie de la buse. Dans cette région, dite région de panache, les forces de flottabilité deviennent dominantes par rapport aux forces d'inertie par conséquent l'allure du profil de la vitesse initiale à la sortie de la buse n'a plus d'effet sur l'écoulement.

Afin de comparer nos résultats à ceux obtenus par Martynenko et al. [3], nous présentons sur la figure 3b, la vitesse verticale modifiée, $U_{\mathrm{cm}}=32 U_{\mathrm{c}} / 3(R e R i)^{0,5}$ en fonction de la distance longitudinale modifiée, $X_{\mathrm{m}}=R i X^{2} / R e$. En effet, il serait fortement important de trouver une solution analytique qui soit valable pour des nombres de Prandtl arbitraires, mais ceci implique de grandes difficultés mathématiques posées par le caractère non-linéaire des équations de conservation [3]. Cependant, une solution approximative peut être construite. En fait, prenant comme base les résultats obtenus pour un nombre de Prandtl égal à 2 et utilisant la méthode des petites perturbations pour différents nombres de Prandtl, il est possible d'obtenir, la vitesse au centre : $u_{\mathrm{c}}=\left(K_{0} / \pi \mu\right)(3 / 8) x^{-1}\left(1+c_{2} \omega x^{2}\right)^{0,5}$ et la température au centre qui s'écrit pour $\operatorname{Pr}=0,71$ sous la forme : $T_{\mathrm{c}}=$ $\left(0,605 Q_{0} / 2 \pi \mu C_{p}\right)(3 / 8) x^{-1}\left(1+2,533 \omega x^{2}\right) /\left(1+3,135 \omega x^{2}\right)$. Les constantes $Q_{0}$ et $K_{0}$ sont déterminées à partir des contraintes d'intégration exprimées à la sortie de la buse [3] (Éq. (6)) et (Éq. (8)), respectivement. Le coefficient $c_{2}$ est fonction du nombre de Prandtl [3], et $\omega x^{2}=G r_{x} / R e^{2}=4 R i X^{2} / R e$ avec $G r_{x}=g \beta Q_{0} x^{2} / \pi C_{p} \rho \nu^{3}$, et $R e=K_{0} / \pi \rho \nu^{2}$. Notons que le nombre de Richardson (ou de Grashof) et le nombre de Reynolds définis par Martynenko et al. [3] sont égaux dans notre étude à $R i R e^{3} X^{2} / 4$ et à $R e^{2} / 4$, respectivement. Les résultats obtenus, pour le cas d'un 

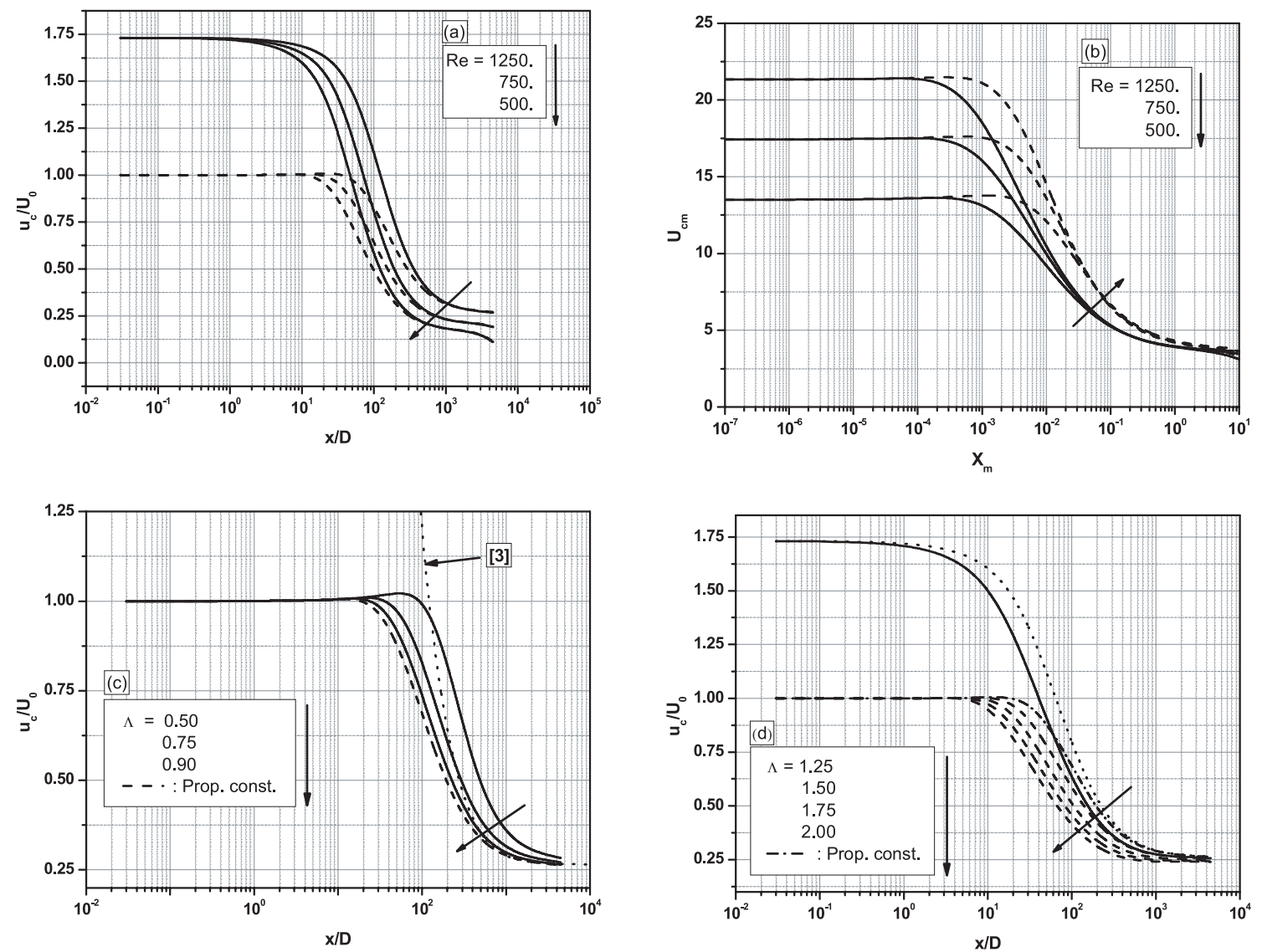

Fig. 3. Évolution de la vitesse au centre, $R i=5 \times 10^{-4}$. (a) Effet du nombre de Reynolds, $(\Lambda=0,75$, trait continu : profil initial de vitesse parabolique, trait interrompu : profil initial de vitesse uniforme). (b) Vitesse au centre modifiée $U_{\mathrm{cm}}$, (trait continu : $\Lambda=0,75$, trait interrompu : $\Lambda=0,5)$. (c) Cas où $\Lambda<1,(R e=1250$, trait interrompu : propriétés constantes, trait pointillé : résultat de Réf. [3]). (d) Cas où $\Lambda>1,(R e=1250$, comparaison avec le cas où les propriétés sont constantes).

profil initial uniforme de la vitesse à la sortie de la buse, figure 3c, ne coïncident avec ceux obtenus par Martynenko et al. [3] que dans la région de panache. La différence est nette dans la région du jet, où les forces d'inertie sont dominantes par rapport aux forces de flottabilité. On constate, d'après les figures $3 \mathrm{a}-\mathrm{d}$ que, pour un profil de la vitesse d'émission uniforme ou parabolique, l'effet de l'écart de température entre le jet et le milieu ambiant n'est pas ressenti dans la zone du noyau potentiel. À la fin du noyau potentiel, début de la décroissance de la vitesse au centre $U_{\mathrm{c}}$, les forces de viscosité deviennent de plus en plus importantes, ce qui entraîne une décélération du jet dans le centre, à cause du développement d'une couche de mélange de part et d'autre du noyau potentiel. La décélération du jet croît avec l'écart de température entre le jet et le milieu ambiant. Nous remarquons, d'après les figures 3c (cas où $\Lambda<1$ ) et $3 \mathrm{~d}$ (cas où $\Lambda>1$ ), qu'il existe une différence entre les résultats obtenus par le présent modèle et ceux obtenus par un modèle à propriétés constantes. Ceci constitue une première correction des résultats obtenus par le modèle de la couche limite, où les propriétés physiques sont considérées constantes.

Sur les figures $4 \mathrm{a}-\mathrm{d}$, on présente l'évolution de la température au centre du jet. Les mêmes observations, effectuées à propos de la vitesse verticale au centre, concernant l'effet de l'écart de température, du profil d'émission et le nombre de Reynolds, figure 4a, sont aussi valables pour la température au centre du jet. Il est à noter que l'analyse du champ de température est moins triviale que pour le cas du champ de vitesse. L'évolution du champ de température résulte de la compétition entre deux phénomènes qui ont un effet opposé. Jusqu'à la fin du noyau potentiel, la vitesse au centre garde une valeur constante et la composante transversale de la vitesse a une valeur positive, ce qui engendre une quantité de chaleur positive transportée par advection dans le sens de l'écoulement. En avançant en aval, loin de la buse la vitesse au centre diminue par contre la composante transversale de la vitesse augmente par valeurs négatives, à cause de l'augmentation de l'entraînement de l'air du milieu ambiant dans le jet, ce qui conduit, d'après la figure $4 \mathrm{c}$, à un phénomène de réchauffement du jet loin de la buse (cas où $\Lambda<1$ ). Par ailleurs, nous remarquons, d'après la figure $4 \mathrm{~d}$, l'existence d'un phénomène de refroidissement du jet loin de la buse, (cas où $\Lambda>1$ ). Le réchauffement ou le refroidissement du jet augmente, pour une section donnée vers la fin du noyau potentiel, en s'éloignant par valeurs décroissantes ou croissantes du cas où les propriétés sont constantes. Afin de comparer 
nos résultats à ceux obtenus par Martynenko et al. [3], nous présentons sur la figure $4 \mathrm{~b}$, la température modifiée au centre, $\theta_{\mathrm{cm}}=8 \theta_{\mathrm{c}} /(R e R i)^{0,5}$ en fonction de la distance longitudinale modifiée, $X_{\mathrm{m}}=R i X^{2} / R e$. Les résultats obtenus, pour le cas d'un profil initial uniforme de la vitesse à la sortie de la buse, figure 4c, ne coïncident avec ceux obtenus par Martynenko et al. [3] que dans la région de panache. La différence est nette dans la région du jet, où les forces d'inertie sont dominantes par rapport aux forces de flottabilité.

\subsubsection{Demi-épaisseur dynamique et thermique}

Sur les figures $5 \mathrm{a}$ et b, on présente l'évolution longitudinale de la demi-épaisseur dynamique et thermique $\mathrm{du}$ jet, respectivement, pour différentes valeurs du paramètre $\Lambda$ (écart de température entre le jet et le milieu ambiant). Nous remarquons, d'après les figures 5a et b, que l'effet de l'écart de température entre le jet et le milieu ambiant est important dans la zone de panache et ceci pour les deux cas de profils de la vitesse à la sortie de la buse. L'élargissement du jet est d'autant plus important pour des valeurs croissantes de l'écart de température. En ce qui concerne l'effet du profil de la vitesse initiale à la sortie de la buse sur ces paramètres, on constate que pour les faibles distances $X$, l'élargissement du jet est plus important pour un profil initial parabolique que pour un profil initial uniforme. Par ailleurs, on remarque que cette différence s'atténue avec la distance $X$, pour disparaître dans la région de panache (grandes distances $X$ ), où les forces de flottabilité sont responsables de l'écoulement.

\subsection{Régime de convection mixte}

\subsubsection{Vitesse et température au centre}

Nous présentons dans cette partie l'évolution des caractéristiques dynamiques et thermiques du jet dans le régime de convection mixte, uniquement pour le cas d'un profil initial uniforme. Nous considérons le cas d'un jet d'air, $\operatorname{Pr}=0,71$ et pour un nombre de Richardson, $R i=0,1$. Les résultats obtenus par le présent modèle sont comparés à ceux du modèle à propriétés constantes et à ceux obtenus par Martynenko et al. [3] .

Pour les faibles distances longitudinales $X$, la vitesse au centre garde une valeur constante jusqu'à la fin du noyau potentiel où les forces d'inertie deviennent prépondérantes par rapport aux forces de flottabilité. Par ailleurs, pour les distances longitudinales très élevées le jet est accéléré sous l'effet des forces de flottabilité qui deviennent dominantes par rapport aux forces d'inertie. L'accélération du jet est plus importante pour les valeurs de $\Lambda$ inférieur à 1 (Fig. 6a). L'analyse du champ de température, figure $6 \mathrm{~b}$, montre le même comportement que celui dans le régime de convection forcée, c'està-dire que la diminution de la température du jet est le résultat de la compétition entre les effets favorable et défavorable des deux composantes longitudinale et
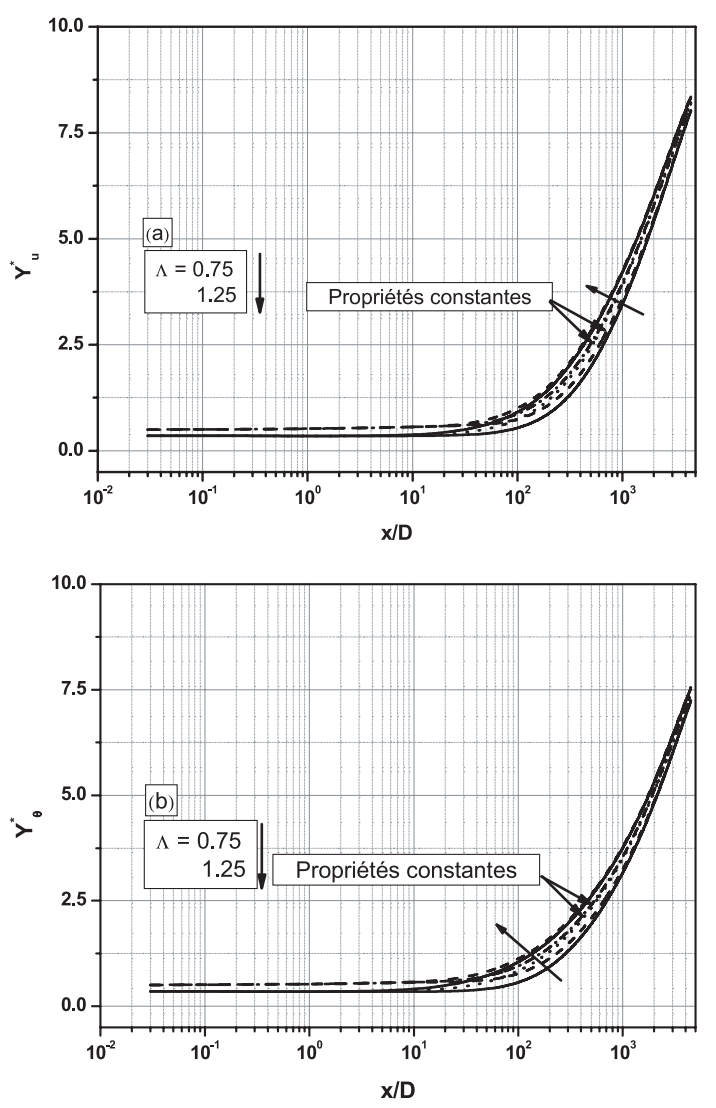

Fig. 5. Évolution de la demi-épaisseur du jet, $R i=5 \times$ $10^{-4}, R e=1250$. (a) Demi-épaisseur dynamique. (b) Demiépaisseur thermique; (trait continu : profil initial de vitesse parabolique, trait interrompu : profil initial de vitesse uniforme).

transversale de la vitesse, respectivement. La comparaison entre nos résultats et ceux obtenus par Martynenko et al. [3] nous permet de constater que le modèle analytique proposé par ces auteurs est valable uniquement dans la zone de panache, où les forces de flottabilité dominent le jet.

\subsubsection{Demi-épaisseur dynamique et thermique}

Sur la figure 7, nous avons représenté l'évolution de la demi-épaisseur dynamique, figure $7 \mathrm{a}$, et thermique, figure $7 \mathrm{~b}$, dans le régime du convection mixte. L'analyse de ces courbes montre que, tout en restant proche de la buse, c'est-à-dire pour les faibles distances $X$, la demi-épaisseur du jet, dynamique ou thermique, garde une valeur constante, indépendamment de l'écart de température entre le jet et le milieu ambiant. Par ailleurs, elle diminue dans la zone de transition pour augmenter linéairement dans la zone de panache. Ceci est dû̀ à une compétition d'effets entre les forces d'inertie et celles de flottabilité qui sont dominantes dans la zone de panache. La diminution de l'élargissement du jet dans la zone de transition est expliquée par le fait que les forces d'inertie et de flottabilité deviennent de même ordre de 

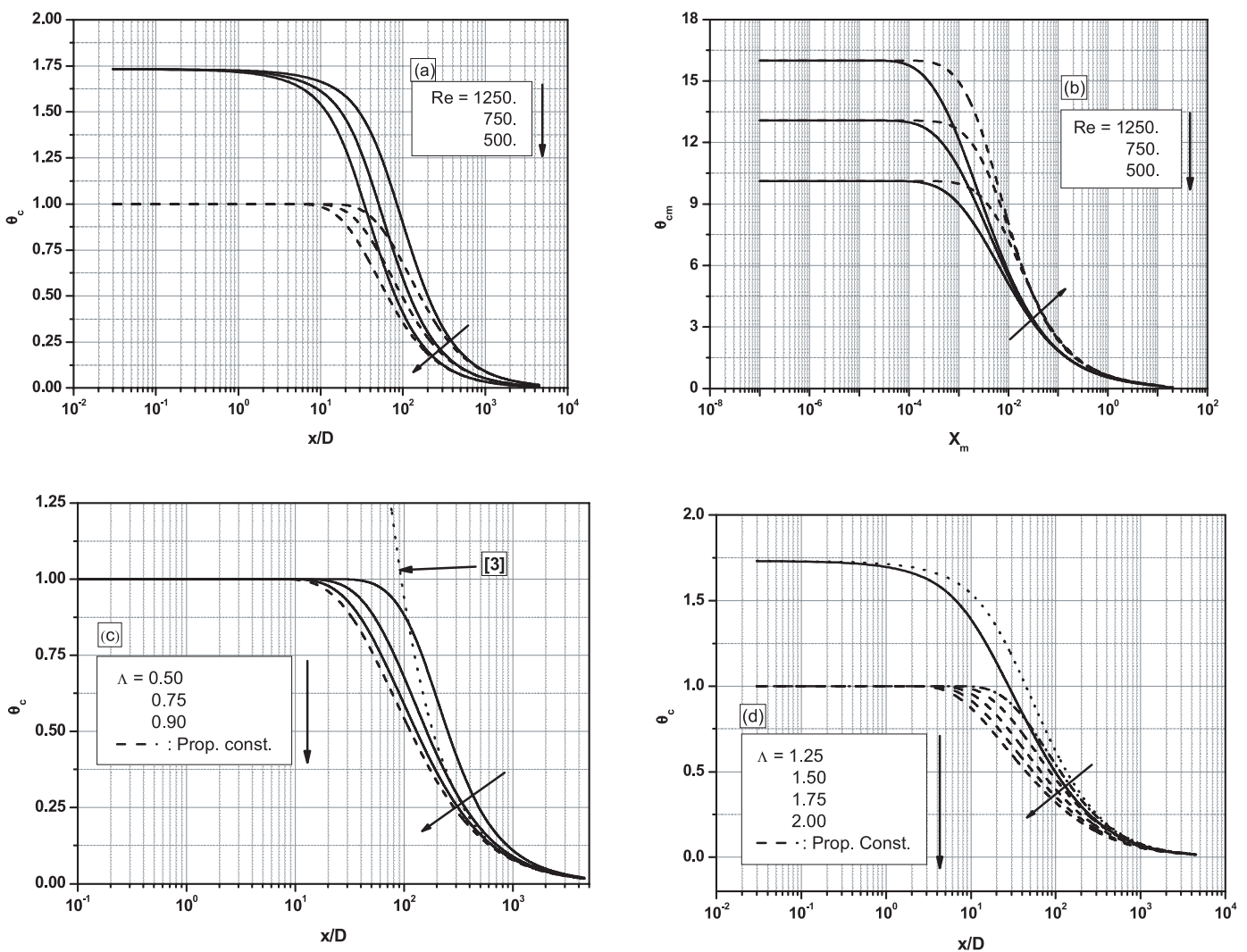

Fig. 4. Évolution de la température au centre, $R i=5 \times 10^{-4}$. (a) Effet du nombre de Reynolds, $(\Lambda=0,75$, trait continu : profil initial de vitesse parabolique, trait interrompu : profil initial de vitesse uniforme). (b) Température au centre modifiée $\theta_{\mathrm{cm}}$, (trait continu : $\Lambda=0,75$, trait interrompu : $\Lambda=0,5)$. (c) Cas où $\Lambda<1,(R e=1250$, trait interrompu : propriétés constantes, trait pointillé : résultat de Réf. [3]). (d) Cas où $\Lambda>1$, $(R e=1250$, comparaison avec le cas où les propriétés sont constantes).

grandeur et leur effet devient comparable, ce qui entraîne une accélération de l'écoulement et par conséquent un rétrécissement du jet. On note aussi que, plus l'écart de température, entre le jet et le milieu ambiant, est grand plus l'expansion du jet est importante et ceci dans les trois régimes de convection. Les profils de la demi-épaisseur dynamique et thermique sont très différents pour les deux cas de profils initiaux à la sortie de la buse : uniforme et parabolique dans la région du jet où les forces d'inertie dominent l'écoulement. Cette différence s'atténue avec la distance $X$, pour disparaître dans la région de panache (grandes distances $X$ ) où les forces de flottabilité sont prépondérantes par rapport aux forces visqueuses.

D'après ce qui précède, on constate que le jet passe du régime de convection forcée au régime de convection mixte à travers un régime intermédiaire, où les forces de flottabilité sont du même ordre de grandeur que les forces d'inertie. En effet, l'intensité des forces de flottabilité visà-vis des forces visqueuses, peut faire passer l'écoulement d'un régime à un autre. Il serait intéressant d'étudier l'effet du nombre de Richardson $G r / R e^{2}$, qui donne une mesure de l'intensité des deux effets, sur la transition du jet du régime de convection forcée au régime de convection mixte et ceci pour différents rapports du paramètre $\Lambda$.

Sur la figure 8, on présente l'évolution de la demiépaisseur, dynamique et thermique, du jet dans différents régimes de convection : le régime de convection forcée, $R i=5 \times 10^{-4}$, le régime de convection mixte, $R i=0,1$ et le régime intermédiaire (de transition). Nous remarquons, d'après la figure 8a, que la demi-épaisseur dynamique est constante dans la zone du jet, pour les faibles valeurs de la distance longitudinale $X$. Dans cette zone le paramètre $\Lambda$ n'a pas d'effet sur l'expansion du jet. Par ailleurs, elle décroît pour atteindre un minimum dans la zone intermédiaire, où les forces d'inertie sont du même ordre de grandeur que les forces de flottabilité. Pour les distances longitudinales $X$ très élevées, la demi-épaisseur du jet augmente rapidement dû à l'augmentation de l'effet des forces de flottabilité qui dominent l'écoulement dans la zone de panache. L'élargissement du jet est plus important pour les faibles valeurs du paramètre $\Lambda$.

\section{Conclusion}

Dans cet article, nous avons présenté une étude numérique des propriétés dynamiques et thermiques d'un écoulement stationnaire de type jet axisymétrique à propriétés variables, en régime laminaire. On s'est intéressé à connaître l'influence de l'écart de température entre le jet et le milieu ambiant, défini par le rapport des températures initiales $\Lambda=T_{0} / T_{\infty}$, sur les caractéristiques du jet dans les deux régimes de convection : forcée et 

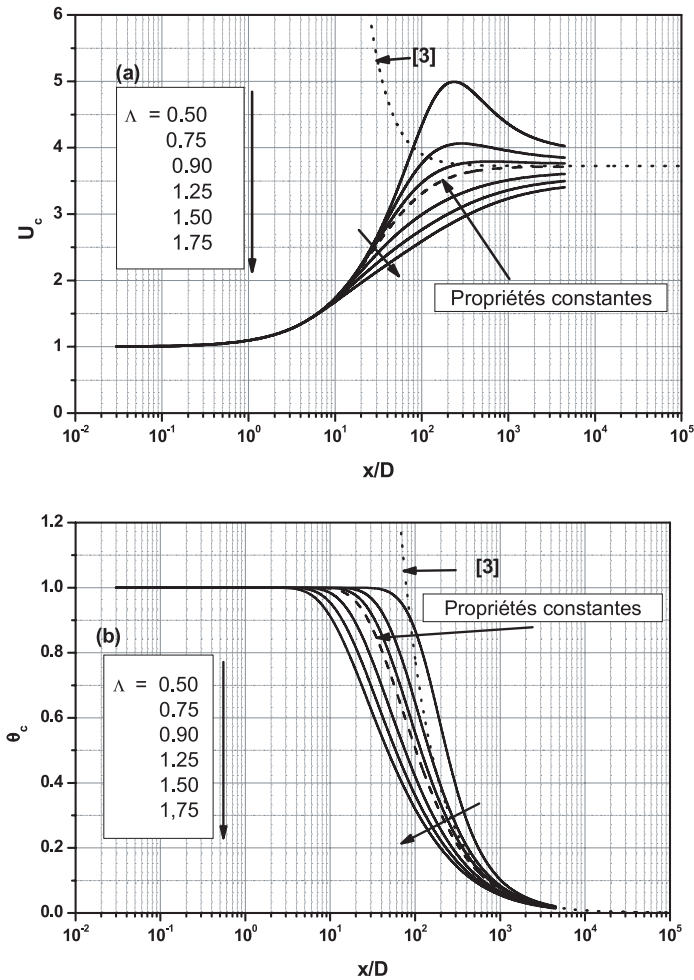

Fig. 6. Vitesse au centre et température au centre, $R i=0,1$, $R e=1250$, profil initial de vitesse : uniforme. (a) Vitesse au centre. (b) Température au centre; (trait interrompu : propriétés constantes, trait pointillé : résultat de Réf. [3]).
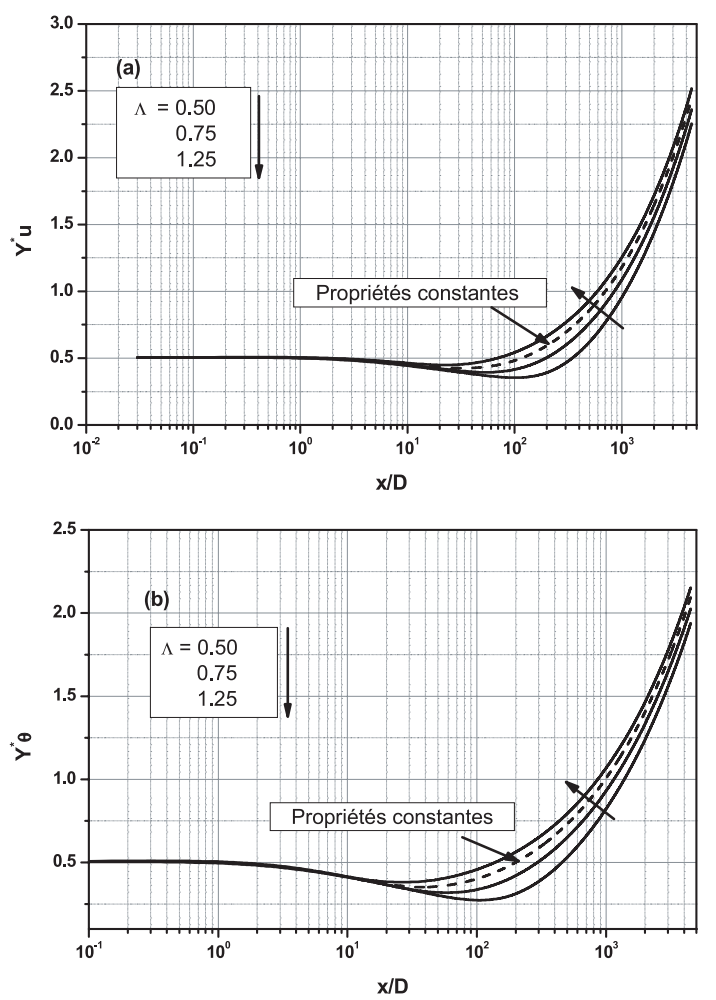

Fig. 7. Évolution de la demi-épaisseur du jet, $R i=0,1, R e=$ 1250. (a) Demi-épaisseur dynamique. (b) Demi-épaisseur thermique; (trait continu : profil initial de la vitesse parabolique, trait interrompu : profil initial de la vitesse uniforme).
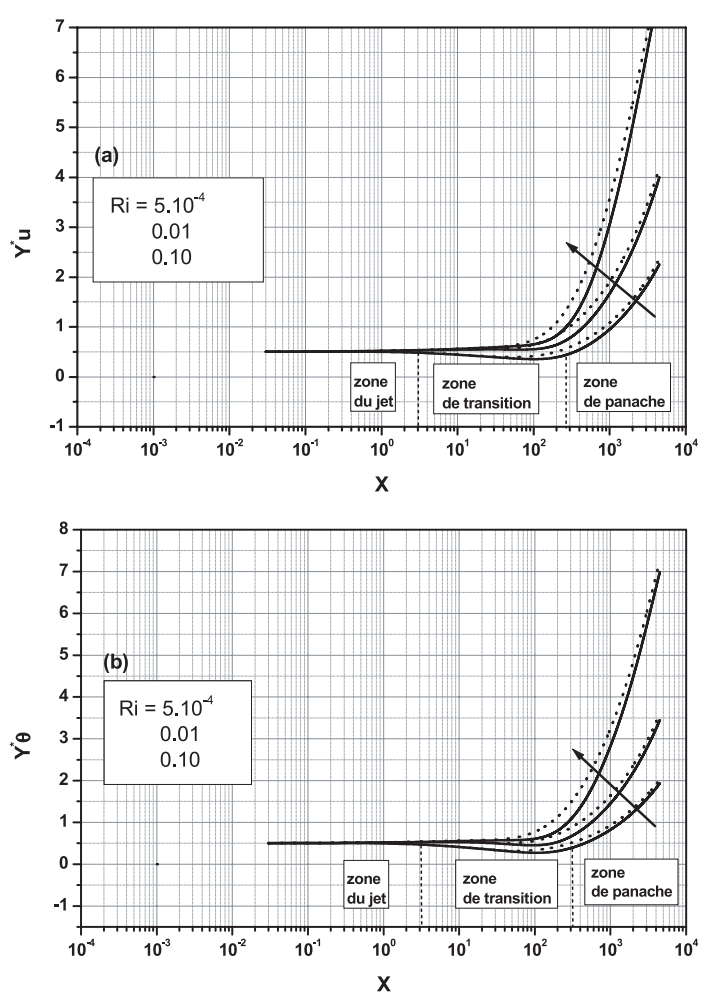

Fig. 8. Évolution de la demi-épaisseur du jet, $R e=$ 1250. (a) Demi-épaisseur dynamique. (b) Demi-épaisseur thermique; (trait continu : $\Lambda=0,5$, trait interrompu : $\Lambda=0,75$ ).

mixte. Une étude de l'effet des conditions d'émission de vitesse et de température à la sortie de la buse est aussi présentée pour les deux cas de profils de vitesse et de température : uniforme et parabolique. Pour la validation de notre code de calcul, les résultats sont comparés à ceux obtenus par Martynenko et al. [3], qui remplacent les conditions d'émission par deux contraintes d'intégration : la conservation de la quantité de mouvement et de l'énergie et qui sont vérifiées pour différents formes de profils de vitesse et de température. Les résultats obtenus par le présent modèle sont aussi comparés à ceux obtenus par le modèle où les propriétés sont constantes.

Pour cela, nous avons considéré les deux cas de l'écart de température entre le jet et milieu ambiant, $\Lambda<1$ et $\Lambda>1$. L'évolution de la vitesse au centre, pour les faibles distances longitudinales, est indépendante de l'écart de température entre le jet et le milieu ambiant, et ceci dans les deux régimes de convection forcée et mixte. Dans cette région les forces d'inertie sont responsables de l'écoulement. Le choix des conditions initiales à la sortie de la buse est surtout important dans la zone du jet, en revanche la variation des propriétés avec la température n'a plus d'effet sur le comportement de l'écoulement. Par ailleurs, on montre que, pour les distances longitudinales très élevées, pour un nombre de Reynolds et un nombre de Richardson fixés, qu'il y a une influence de plus en plus significative de $\Lambda$, sur les grandeurs dynamiques et thermiques du jet. En effet, dans la zone de panache les forces de flottabilité deviennent prépondérantes par 
rapport aux forces d'inertie, la variation des propriétés avec la température a un effet important. Par contre, le choix des conditions initiales à la sortie de la buse est ignoré. La validation du modèle, présenté dans cette étude, est réalisée en se référant aux résultats obtenus par Martynenko et al. [3]. Nos résultats et ceux obtenus par ces auteurs montrent une bonne concordance dans la zone de panache.

Pour des rapports $\Lambda$, qui correspondent à un écart de température entre le jet et le milieu ambiant, nous avons montré que : pour les rapports $\Lambda<1$, le jet subit un échauffement d'autant plus important que ce paramètre est plus faible, alors que, pour le cas des rapports $\Lambda>1$, le jet est le siège d'un phénomène de refroidissement. L'évolution de la demi-épaisseur du jet, dynamique et thermique, présente un comportement indépendant du paramètre $\Lambda$, surtout dans la zone du jet (faibles distances longitudinales $X$ ). Pour les grandes distances longitudinales $X$, l'évolution de la demi-épaisseur du jet est influencée fortement par l'écart de température. En effet, on a montré que cette grandeur augmente dans la zone de panache en passant par un minimum dans la zone de transition, dû à une augmentation de l'effet des forces de flottabilité. L'effet de la variation des propriétés sur le champ de température est surtout marqué par une diminution vers la fin du noyau potentiel, dû à l'augmentation de la composante transversale de la vitesse, par valeurs négatives, qui transporte une quantité de chaleur dans le sens contraire de l'écoulement.

\section{Références}

[1] H. Schlichting, Laminare strahlausbreitung, ZAMM, 13, 260, Boundary layer theory, Co. McGraw-Hill, 1933, pp. $164-181$

[2] W. Bickley, The plane jet, Phil. Mag. 23 (1937) 727

[3] O.G. Martynenko, V.N. Korovkin, Yu.A. Sokovishin, The class of self-similar solutions for laminar buoyant jets, Int. J. Heat Mass Tran. 32 (1989) 2297-2307

[4] W.S. Yu, H.T. Lin, H.C. Shih, Rigorous numerical solutions and correlations for two-dimensional laminar buoyant jets, Int. J. Heat Mass Tran. 35 (1992) 11311141

[5] L. Landau, See L.D. Landau, E.M. Lifshitz, Fluid Mechanics, Pergamon Press, Oxford, 1943, p. 86

[6] O.G. Martynenko, V.N. Korovkin, Numerical investigation of turbulent plane and buoyant jets, Int. J. Heat Mass Tran. 35 (1992) 635-639
[7] J.C. Mollendorf, B. Gebhart, Thermal Buoyancy in round Laminar Vertical Jets, Int. J. Heat Mass Tran. 16 (1973) $735-745$

[8] R.S. Brand, F.J. Lahey, The heated laminar vertical jet, J. Fluid Mech. 29 (1967) 305-315

[9] J.W. Yang, R.D. Patel, Effect of Buoyancy on forced convection in a two dimensional wall jet along a vertical wall, J. Heat Transfer 95 (1973) 121-123

[10] H. Ben Aissia, Y. Zaouali, S. El Golli, Numerical study of the influence of dynamic and thermal exit conditions on axisymmetric laminar bouyant jet, Numerical Heat Transfer, Part A 42 (2002) 427-444

[11] H. Ben Aissia, Étude numérique et expérimentale par imagerie et anémomètre laser doppler d'un jet axisymétrique, thèse, Université El Manar II, Tunisie, 2002

[12] M.L. Albertson, Jun. Asce, Y.B. Dai, R.A. Jensen, H. Rouse, M. Asce, Diffusion of submerged jets, Am. Soc. civil engineers 74 (1948) 1571-1596

[13] W.M. Pitts, Effects of global density and Reynolds number variations on mixing in turbulent axisymmetric jets, Rapport NBSIR 86-3340, Department of commerce, Washington, 1986

[14] A.D. Birch, D.R. Brown, H.G. Dodson, J.R. Thomas, The turbulent concentration field of a methane jet, J. Fluid Mech. 88 (1978) 431

[15] L. Fulachier, R. Borghi, F. Anselmet, P. Paranthoen, Influence of density variations on the structure of lowspeed turbulent flows, J. Fluid Mech. 203 (1989) 577-593

[16] D.R. Dowling, P.E. Dimotakis, Similarity of the concentration field of gas phase turbulent jets, J. Fluid Mech. 218 (1990) 109-141

[17] J.P.H. Sanders, B. Sarh, I. Gokalp, Variable density effects in axisymmetric isothermal turbulent jets: a comparison between a first and a second order turbulence model, Int. J. Heat Mass Transfer 40 (1997) 823-842

[18] J.P. Sanders, B. Sarh, I. Gokalp, Étude numérique des jets turbulents à température élevée, Review of general thermodynamics 35 (1996) 232-242

[19] W.M. Kays, M.E. Crawford, Convective heat and mass transfer, Co. McGraw-Hill Book, New York, 2nd Ed, 1980

[20] C. Fonade, Cours. Étude des jets, Application à la fluidique, Institut national polytechnique de Toulouse, France, 1967

[21] A.M. Dalbert, F. Penot, J.L. Peube, Convection naturelle laminaire dans un canal vertical chauffé à flux constant, Int. J. Heat Mass Tran. 24 (1981) 1463-1473

[22] C.A.J. Fletcher, Computational techniques for fluid dynamics 1, Fundamental and general techniques, Co. Springer-Verlag, Berlin Heidelberg, 2nd Ed., 1991 